ENCYCLOPEDDIE Encyclopédie berbère

BERBERE

$14 \mid 1994$

14 | Conseil - Danse

\title{
Dahir berbère
}

(16 mai 1930)

\section{G. Lafuente}

\section{OpenEdition}

Journals

Édition électronique

URL : http://journals.openedition.org/encyclopedieberbere/2361

DOI : 10.4000/encyclopedieberbere.2361

ISSN : 2262-7197

\section{Éditeur}

Peeters Publishers

\section{Édition imprimée}

Date de publication : 1 septembre 1994

Pagination : 2178-2192

ISBN : 2-85744-741-8

ISSN : 1015-7344

\section{Référence électronique}

G. Lafuente, "Dahir berbère », Encyclopédie berbère [En ligne], 14 | 1994, document D05, mis en ligne le

01 mars 2012, consulté le 10 décembre 2020. URL : http://journals.openedition.org/

encyclopedieberbere/2361 ; DOI : https://doi.org/10.4000/encyclopedieberbere.2361

Ce document a été généré automatiquement le 10 décembre 2020.

(c) Tous droits réservés 


\section{Dahir berbère}

(16 mai 1930)

\section{G. Lafuente}

1 Le 16 mai 1930, la France promulguait au Maroc un décret devenu célèbre : « Le Dahir Berbère ». Celui-ci fut rapidement considéré par tous les observateurs comme le catalyseur du nationalisme marocain, alors qu'il n'était, aux yeux des Français, qu'un dahir parmi tant d'autres. Cependant, les conditions politiques, économiques, psychologiques qui prévalaient lors de sa promulgation contribuèrent à susciter des réactions qui dépassèrent par leur violence tout ce que pouvaient en attendre les juristes qui l'élaborèrent, tout comme les Marocains qui s'y opposèrent.

2 Ce Dahir avait pour but l'adaptation de la « Justice Berbère » aux conditions propres de l'époque et, de ce fait, correspondait à l'esprit de la politique inaugurée au Maroc par Lyautey quand il signa le Dahir du 11 septembre 1914. La caractéristique fondamentale de cette politique consistait à préserver l'autonomie traditionnelle des Berbères, essentiellement dans le domaine juridique, en les soustrayant à la législation islamique ou « Chrâa ", et en maintenant leur droit Coutumier ORF ou IZREF. Elle reconnaissait et garantissait l'application des lois coutumières berbères, mais sans préciser la nature de ces lois, ni stipuler quelles étaient les tribus dites «berbères". Ce fut le rôle de l'administration de régler ces deux problèmes, et de déclarer «berbères » les tribus qui se soumettaient aux autorités militaires. Il faut rappeler que la population du Maroc, à cette époque, était considérée comme étant composée dans sa grande majorité de Berbères (plus des 3/4). Dans les premières années du Protectorat, cette politique ne provoqua aucune réaction, car elle entérinait un état de fait qui avait toujours existé. Cette politique, jusqu'en 1925, fit l'objet d'un grand nombre de dahirs et d'arrêtés viziriels destinés à la préciser et à en fixer les modalités d'application.

3 L'étude des groupements berbères commença dès les premières années du Protectorat, le 9 janvier 1915, quand fut créé à Rabat le : "Comité d'Etudes Berbères », dans le but de : «... centraliser les travaux établis dans les différentes régions sur les populations berbères du Maroc et d'en retirer des résultats pratiques concernant l'organisation et l'administration des tribus ». Ce Comité publia les résultats des recherches entreprises 
dans la revue Archives Berbères, qui constituent un fonds extrêmement riche pour la connaissance de ce monde berbère.

Mais, au fur et à mesure que les enquêtes progressaient, certains observateurs firent remarquer la persistance chez les Berbères de superstitions animistes fort peu orthodoxes, de rites païens pré-islamiques totalement contraires aux règles de l'Islam, ainsi que leur refus des règles du "Chrâa", notamment dans le domaine du statut personnel (mariage, divorce, héritage), ce qui n'empêchait pas ces mêmes Berbères d'affirmer qu'ils étaient de vrais musulmans. De là à en déduire que leur attachement à l'Islam était superficiel, il n'y avait qu'un pas qui fut vite franchi.

5 En conséquence, les tenants de l'assimilation pensèrent que si les Berbères pouvaient être préservés de toute influence arabe - donc musulmane - il serait possible d'en faire des «Français" par le canal des juridictions françaises, des écoles françaises et de la religion chrétienne... ! De plus, il ne fallait pas oublier la « ressemblance " physique existant entre les Berbères et les paysans français, vu que les premiers: «... sont, comme nous, issus de la race aryenne ».

De telles affirmations ne pouvaient que choquer les Marocains et augmenter leur inquiétude, d'autant plus qu'elles émanaient d'hommes proches de la Résidence, chargés d'importantes fonctions. Il était donc normal que ces propos fussent considérés par les Marocains comme exprimant les tendances officielles de la politique française au Maroc Ils ont voulu la faire connaître dans tout le monde musulman, et en ont traduit l'essentiel qu'ils ont fait paraître dans la presse du Moyen Orient.

7 De toute la «littérature " consacrée au monde berbère, c'est peut-être le livre du commandant Paul Marty (Le Maroc de Demain, 1925), qui exprimait de la façon la plus claire et la plus officielle ce que devrait être la politique berbère du Protectorat, et ce, avec d'autant plus d'assurance qu'il se plaçait sous les auspices du Général Lyautey et de l'orientaliste Louis Massignon. L'assimilation des Berbères se ferait donc grâce aux écoles franco-berbères, dont il définit ainsi la forme et la fonction : « ... l'école francoberbère c'est donc l'école française par l'enseignement et la vie, berbère par les élèves. Donc, pas d'intermédiaire étranger. Tout enseignement de l'arabe, toute intervention du "fquih", toute manifestation islamique seront rigoureusement écartés... En résumé, ces écoles berbères seront autant des organismes de politique française et des instruments de propagande que des centres pédagogiques proprement dits ».

8 La réalisation d'un tel plan avait commencé en 1923, et on avait demandé à Louis Massignon de superviser l'installation de ces écoles. Mais les résultats ne furent pas à la hauteur de ces ambitions, et en 1930, 20 écoles avaient été créées et elles ne scolarisaient que 700 élèves.

9 La promulgation, le 16 mai 1930, du Dahir Berbère, s'inscrit donc dans la droite ligne de cette politique, et, dans l'esprit de ceux qui l'élaborèrent, il en marque, sinon l'aboutissement, du moins une étape importante.

\section{L'Élaboration du Dahir}

$10 \mathrm{Au}$ fur et à mesure que la pacification s'étendait le nombre des tribus berbères augmentait et elles se voyaient appliquer le Dahir de 1914 qui reconnaissait leurs « lois et coutumes propres ", en vertu desquelles elles devaient être administrées. 
11 Selon les coutumiers alors en vigueur, l'administration des tribus berbères fut confiée aux « djemaas ", assemblées où se trouvaient réunis les notables et les " anciens » qui connaissaient le mieux les lois de leurs fractions. En vérité, il s'agissait là encore d'un fiction, car très rapidement, les membres de la djemaa furent choisis par les administrateurs français. A ce moment là, ces djemaas n'avaient aucune attribution judiciaire, et la justice était rendue par un arbitre choisi par les parties en conflit. Si les parties ne pouvaient se mettre d'accord sur le choix de cet arbitre, c'était la djemaa qui le désignait. Toutefois, le libre choix d'un tel personnage ne pouvait plaire bien longtemps aux autorités françaises de contrôle qui préféraient - et de loin - que la justice fût rendue par la djemaa, dont les membres étaient désignés par ces mêmes autorités...!

Pour une réglementation plus précise, la Direction des Affaires Indigènes créa une commission d'étude de la justice berbère dont l'objet était de déterminer les règles de compétence et de procédure des djemaas judiciaires en tribu de coutume berbère. Cette commission ajouta aux compétences déjà reconnues des djemaas judiciaires, la possibilité de trancher en matière civile et commerciale, ce qui était en fait du ressort du Caïd ou du Pacha. Les Caïds ne gardaient que la répression des affaires pénales. En matière criminelle, le Caïd devait en référer au Haut Tribunal Chérifien, qui jugeait, en principe, d'après la coutume locale. Toutefois il faut noter une exception qui sera lourde de conséquences : le monde berbère, n'étant pas totalement " pacifié ", était soumis, non pas aux autorités civiles, mais à un commandement militaire. De ce fait, certains crimes, notamment ceux commis à l'encontre de colons ou de soldats français étaient jugés par un tribunal militaire français qui avait le « droit » d'appliquer la peine de mort. Ces raisons de sécurité seront invoquées plus tard pour justifier l'article $6 \mathrm{du}$ dahir du 16 mai 1930.

On le voit, dès 1924, - Lyautey est encore Résident Général -, le problème de la justice en pays berbère est parfaitement posé, mais non encore réglé selon le vœu des autorités françaises.

Fin 1929, 81 djemaas judiciaires existaient pour l'ensemble des tribus classées «berbères » et elles inspiraient une confiance absolue. Tout aurait été pour le mieux si une grave lacune, pour ne pas dire une lacune fondamentale dans la poursuite de cette politique, n'était venue en perturber le déroulement. En effet, issues de simples circulaires administratives, les djemaas ne reposaient sur aucun fondement légal. Qui plus est, ces mesures administratives n'avaient pas été contresignées par les Autorités Marocaines.

Il aurait fallu pour cela qu'un dahir - signé du Sultan - entérinât la création de ces djemaas judiciaires. Or, Moulay Youssef était intraitable sur ce sujet, et cette absence de légitimité légale entraînait l'absence de l'autorité de la chose jugée à l'égard des autres juridictions, ainsi que l'absence d'obligation juridique d'exécuter la sentence. Certes, au sein des tribus à qui ce système donnait entière satisfaction, il n'y avait pas de contestation. Mais avec la pénétration des colons, hommes d'affaires, commerçants, le danger se précisait. C'est ainsi qu'il fut impossible aux tribunaux français de reconnaître une valeur juridique aux sentences des djemaas pourtant créées par les autorités françaises... ! Il s'ensuivit une crise de confiance grave dans le monde berbère envers l'institution, mais aussi envers ceux qui l'avaient soutenue, à tel point que de nombreux officiers des Affaires Indigènes refusèrent d'étendre l'expérience. Il fallait absolument 
donner aux djemaas judiciaires une existence légale, et ce, par un dahir scellé du Sultan.

Il était donc impératif de rendre légaux tous les actes et jugements rendus par les djemaas judiciaires, mais cette nécessité se heurtait à un écueil de taille incontournable jusqu'alors: le refus du Sultan d'apposer son sceau au bas d'un texte qui ne reconnaîtrait pas la prééminence absolue du Chrâa sur toute autre législation. Or, en cette année 1930, la situation au Maroc avait changé. Le 17 novembre 1927, le Sultan Moulay Youssef était décédé, et il avait été remplacé sur le trône Alaouite par son troisième fils, Sidi Mohammed, le futur Mohammed V, un jeune homme de 18 ans. Il était donc normal de penser que, vu son inexpérience, il serait plus malléable, et qu'il scellerait - enfin - le dahir officialisant les djemaas judiciaires.

D'autre part, les responsables politiques français ressentaient l'urgence de consolider leur politique berbère devant une extension - certes diffuse - mais de plus en plus importante des sentiments nationalistes, exacerbés par une situation économique difficile.

18 C'est dans ce contexte et dans cet esprit qu'un arrêté résidentiel créait le 7 décembre 1929, une fois de plus, une Commission chargée de l'étude de l'organisation de la justice dans les tribus de coutume berbère. Les 14 membres de cette Commission avaient pour tâche de proposer au Gouvernement toutes suggestions utiles concernant le fonctionnement de la Justice Berbère ainsi qu'un projet de Dahir, qui, revêtu du sceau du Sultan, légaliserait l'existence des Djemaas et la valeur de leurs jugements.

19 Très vite, deux thèses se sont affrontées : celle des avocats par la voix de M. Picard, et celle du Général Noguès et du Cabinet Militaire.

Pour le Bâtonnier de l'ordre des Avocats de Rabat, Mr Picard, le but poursuivi était tout simplement de créer un tribunal français, c'est à dire « ... un juge de paix assisté au non d'assesseurs berbères parfaitement au courant de l'Orf ». Et M. Picard terminait son intervention en déclarant : «Ce que nous avons réalisé en Algérie en 1874, nous devons en 1930 pouvoir le réaliser au Maroc où le Berbère, pourvu qu'on lui applique sa coutume, ne demande pas mieux d'être jugé par un Français indifférent à toutes les influences.»

20 A cette thèse fut opposée celle de la Direction des affaires indigènes. C'est ainsi que le Général Noguès, tout en reconnaissant l'efficacité des djemaas judiciaires fut très réticent quant à l'introduction d'un juge français au sein d'un tribunal berbère. Une telle réforme ne lui paraissait pas opportune. Si, en revanche, on donnait un statut légal aux djemaas, tout le monde serait satisfait, le gouvernement voulant: « ... consacrer ce qui existe $»$.

21 C'est donc dans cette perspective que se poursuivirent les débats, et ils aboutirent à un projet de Dahir qui reconnaissait la compétence des djemaas en matière civile, commerciale, mobilière et immobilière, ainsi que celle des chefs de tribu en matière pénale. Ce même Dahir visait aussi à soustraire la justice berbère à l'ingérence du haut tribunal chérifien qui jugeait en fonction du Chrâa. Le texte en fut distribué à tous les membres de la commission pour qu'ils puissent y réfléchir avant la réunion suivante qui se tint le 6 mars 1930. Il est évident qu'un tel projet ne comportait aucun aspect susceptible de provoquer les critiques, et la signature du Sultan n'aurait, semble-t-il, soulevé aucun problème. 
Que l'on sache par la présente, que notre Majesté Chérifienne,

Considérant que le dahir de notre Auguste père, S.M. le Sultan Moulay Youssef, en date du 11 septembre 1914 a prescrit dans l'intérêt du bien de nos sujets et de la tranquillité de l'Etat de respecter le statut coutumier des tribus berbères pacifiées..., qu'il devient opportun de préciser aujourd'hui les conditions particulières dans lesquelles la justice sera rendue dans les mêmes tribus :

A décrété ce qui suit :

Art. 1

Dans les tribus de Notre Empire reconnues comme étant de coutume berbère, la répression des infractions commises par des sujets marocains qui serait de la compétence des caïds dans les autres parties de l'Empire, est de la compétence des chefs de tribu.

Pour les autres infractions, la compétence et la répression sont réglées par les articles 4 et 6 du présent dahir.

Art. 2

Sous réserve des règles de compétence qui régissent les tribunaux français de Notre Empire, les actions civiles ou commerciales, mobilières ou immobilières sont jugées, en premier ou dernier ressort, suivant le taux qui sera fixé par arrêté viziriel, par les juridictions spéciales appelées tribunaux coutumiers. 
Ces tribunaux sont également compétents en toute matière de statut personnel ou successoral.

Ils appliquent, dans tous les cas, la coutume locale.

Art. 3

L'appel des jugements rendus par les tribunaux coutumiers, dans les cas où il serait recevable, est porté devant les juridictions appelées tribunaux d'appel coutumiers.

Art. 4

En matière pénale, ces tribunaux d'appel sont également compétents, en premier et dernier ressort, pour la répression des infractions prévues à l'alinéa 2 de l'article premier ci-dessus, et en outre de toutes les infractions commises par des membres des tribunaux coutumiers dont la compétence normale est attribuée au chef de la tribu.

Art. 5

Auprès de chaque tribunal coutumier de première instance ou d'appel est placé un commissaire du Gouvernement, délégué par l'autorité régionale de contrôle de laquelle il dépend. Prés de chacune de ces juridictions est également placé un secrétairegreffier, lequel remplit en outre les fonctions de notaire.

Art. 6

Les juridictions françaises statuant en matière pénale suivant les règles qui leur sont propres, sont compétentes pour la répression des crimes commis en pays berbère quelle que soit la condition de l'auteur du crime.

Dans ces cas est applicable le dahir du 12 août 1913 (9 ramadan 1331) sur la procédure criminelle.

Art. 7

Les actions immobilières auxquelles seraient parties, soit comme demandeur, soit comme défendeur, des ressortissants des juridictions françaises, sont de la compétence de ces juridictions.

Art. 8

Toutes les règles d'organisation, de composition et de fonctionnement des tribunaux coutumiers seront fixés par arrêtés viziriels successifs, selon les cas et suivant les besoins.

Sept jours plus tard, le 13 mars 1930, la Commission se réunit pour la troisième et dernière fois. Il s'agissait de discuter le nouveau dahir, et il est évident que c'est l'article 6 qui retint l'attention des membres présents car ils étaient conscients des conséquences qu'il impliquait.

D'entrée, M. Benazet fit remarquer qu'il faudrait s'attendre aux réticences du Maghzen devant une telle atteinte à ses droits, et $\mathrm{M}$. Blanc, fidèle à son respect du traité de Protectorat et aux prérogatives attachées à la fonction de chef religieux du Sultan, ne put que réitérer ses doutes quant à l'adhésion de ce dernier au texte qui lui serait soumis.

Devant ces réserves, le Président Cordier invoqua le principe de la sécurité des gens et la nécessité de faire face à tous les délits graves dans les zones non encore "pacifiées ». Mais l'objection fondamentale persistait, et c'est le Commandant Marty qui l'exprimera d'une façon très pertinente: «Les objections que peut élever le Maghzen sont du domaine religieux. Le Sultan en sa qualité d'Imam ne peut consentir à décréter lui même qu'une partie de ses sujets musulmans n'obéira pas à la loi révélée ». Cet argument sera repris et amplifié par tous les opposants au dahir. 
Le Président Cordier passa outre et, jouant les Ponce Pilate, conclura : «... Nous avons étudié le problème comme techniciens. Il échappe à la Commission de l'aborder du point de vue gouvernemental. Il appartient au Gouvernement de décider ».

Le texte du dahir fut présenté au jeune Sultan Mohammed Ben Youssef âgé alors d'une vingtaine d'années, par le Résident Général, M. Lucien Saint. Il est très difficile, pour ne pas dire impossible, de déterminer quels furent les arguments invoqués par le Résident pour convaincre son interlocuteur. Il n'en demeure pas moins que c'est le représentant de la France qui porte l'entière responsabilité du Dahir du 16 mai 1930, tant il semble évident que le futur Mohammed V ne pouvait saisir à ce moment toutes les implications juridiques, morales et religieuses du texte au bas duquel il allait apposer son sceau.

En fait, il est évident que l'autorité du Sultan dans le domaine judiciaire, et les prérogatives qui étaient les siennes en tant qu'Imam, avaient été singulièrement écornées, ne serait-ce que par le Dahir de 1914 qui, de la façon la plus officielle, soustrayait à la justice sultanienne près des $3 / 4$ de ses sujets. De plus, durant les 16 années qui précédèrent le dahir du 16 mai, de très nombreux textes et arrêtés viziriels concernant le monde berbère furent promulgués, sans que personne ne songeât à émettre la moindre réserve. Il faut bien comprendre que le sentiment nationaliste était encore très diffus, et que de 1912 à 1930, il était très difficile pour les Marocains de protester contre un dahir quelconque, ceux-ci étant tous signés par le Sultan.

32 Mais les esprits avaient évolué, et il ne fait aucun doute que ce texte, dans le contexte de l'année 1930, était l'erreur qu'attendaient les chefs nationalistes, erreur qu'il fallait exploiter.

33 Signé le 16 mai par le Sultan, et promulgué le 23 du même mois par le Résident Général, M. Lucien Saint, c'est l'article 6 qui mit le feu aux poudres. Il prévoyait que : «... Les juridictions françaises statuant en matière pénale suivant les règles qui leur sont propres sont compétentes pour la répression des crimes commis en pays berbère, quelle que soit la condition de l'auteur du crime ».

4 Cet article fut interprété par les nationalistes marocains comme une violation de la religion musulmane, pourtant protégée par le traité de Fès de 1912, dans la mesure où il soustrayait à la justice islamique toutes les tribus berbères où se trouvaient la majorité des Marocains, et par le fait même, amoindrissait les pouvoirs du Sultan. Si l'on ajoute à cela le fait que l'élite des grandes villes connaissait parfaitement les buts visés par l'installation des écoles franco-berbères, on comprend que ses membres aient vu dans cet article une tentative de la France pour accroître son emprise sur le Maroc.

5 Ce Dahir, au grand étonnement des Français, fut considéré certes comme une attaque contre l'Islam mais aussi comme un «complot » devant permettre l'évangélisation du Maroc. Ces deux aspects du problème furent pratiquement les seuls portés à la connaissance des Musulmans du Moyen-Orient dans la tapageuse campagne de presse qui s'ensuivit. Il faut y voir la conjonction de plusieurs facteurs, et tout d'abord l'évolution entre 1915 et 1930 de la jeunesse intellectuelle marocaine et plus particulièrement de celle de Fès.

\section{La Société marocaine et son évolution : 1915-1930}

Les années 1925-1930 furent une période de fermentation intellectuelle dans l'ensemble du Monde Musulman. Au Maroc, on assista à la création des «Ecoles Libres » qui 
répandirent dans la jeunesse les idées réformatrices salafistes. L'expansion de la Salafiya - qui entendait trouver la solution aux problèmes du monde arabe par un emploi de la technique moderne mise au profit de la restructuration des fondements de l'Islam - prépara les esprits à un retour à la culture nationale et aux traditions, particulièrement en matière religieuse. Enfin des "Sociétés Secrètes " à Fès, Rabat, Tétouan recrutèrent leurs membres parmi les enseignants des «Ecoles Libres» qui deviendront les dirigeants du mouvement nationaliste marocain.

Toute une intelligentsia était prête à réagir pour peu qu'on lui en donnât le prétexte. Il fallait un événement exceptionnel, un catalyseur, pour faire la synthèse de tous ces sentiments refoulés, de toutes ces espérances non exprimées, pour unifier tous ces clubs informels et encore embryonnaires. Le Dahir Berbère, promulgué le 16 mai 1930, fut ce catalyseur, mais encore fallait-il une idée-force qui pût rassembler autour d'elle tous ces mécontents, un dénominateur commun de toute une jeunesse avide d'action. Une « atteinte » à la religion musulmane fut ce dénominateur.

\section{La « Croisade » contre I'Islam ?}

Cette « atteinte » à l'Islam ne manqua pas d'étonner les autorités du Protectorat, car, en effet, rien, et il faut le souligner, ne pouvait accréditer l'idée que la France voulait d'une manière ou d'une autre s'immiscer dans les affaires religieuses des Marocains. Mais, certains événements ne pouvaient qu'exacerber la sensibilité des Marocains et provoquer leur exaspération pour peu qu'ils eussent lieu dans un climat psychologique propice.

39 C'est ainsi que cette année 1930 fut celle des festivités grandioses accompagnant la célébration du centenaire de la présence française en Algérie. Préparées dès le mois de Janvier, elles culmineront le 14 juin par une manifestation à Sidi Ferruch, où, cent ans auparavant les troupes du Général de Bourmont avaient débarqué. Peut-être encore plus traumatisante fut la tenue à Carthage, du 7 au 11 mai 1930, d'un Congrés Eucharistique, dans une solennité quelque peu extravagante. Des milliers de jeunes Français parcoururent les rues de la ville, affublés de vêtements semblables à ceux que portaient les croisés... ! Les Musulmans, en Tunisie et ailleurs, y virent la célébration de la victoire de la « Croix » sur le « Croissant ».

Cette peur que les Musulmans, qu'ils fussent Tunisiens, Algériens, ou Marocains, éprouvaient devant les activités des représentants de l'Eglise Chrétienne est très significative du climat affectif et émotionnel dans lequel ils vivaient, bien que, officiellement, rien ne pouvait alimenter leurs craintes. Dans ce contexte très particulier, où les Marocains - surtout ceux de Fès, capitale religieuse du Royaume - se sentaient agressés dans leur foi, un événement, en soi minime, mais dont l'impact fut considérable sur les esprits et les cœurs, frappa durement les habitants de la ville d'où partira en 1930, le mouvement de protestation contre le dahir Berbère.

41 Deux années auparavant, une jeune fassi, Mohammed Abdeljalil, fils d'une des plus grandes familles de la ville demanda à embrasser le Christianisme. Non seulement un musulman apostasiait - acte passible de la peine de mort selon le Chrâa -, mais Mohammed Abdeljalil entra en 1929 dans l'Ordre Franciscain, et fut ordonné prêtre en 1935 sous le nom de Jean Mohammed Abdeljalil. Dès qu'elle fut connue, cette conversion fut ressentie comme une honte et une profonde humiliation par toute la 
communauté fassie, mais tout autant, si ce n'est plus, comme la preuve de l'efficacité de la mission évangélisatrice menée au Maroc par l'église catholique avec la complicité de la Résidence.

Mais la «bête noire » des nationalistes fut incontestablement la revue mensuelle Le Maroc Catholique, patronnée par l'archevêque de Rabat, Mgr Vieille. On comprend parfaitement que les lecteurs marocains fussent ulcérés par les affirmations que l'on y trouvait, d'autant plus que les auteurs de ces textes ne considéraient pas leurs idées comme utopiques, mais comme parfaitement réalisables. Un grand nombre d'extraits furent traduits et publiés dans la presse du Moyen-Orient dans le cadre des protestations organisées contre le Dahir, pour bien prouver que le but des Français du Maroc était de : « ... faire sortir les Berbères de l'enceinte de l'Islam ».

Cette même revue publiera, en 1927, le compte-rendu de l'Assemblée Générale des pères Blancs tenue à Alger, sous un titre non équivoque "L'évangélisation des Berbères ». Constatant tout d'abord l'erreur commise en Algérie en imposant une justice musulmane ainsi que la langue arabe à des Berbères régis par leurs coutumes, les auteurs du texte se félicitent de voir que : «... Le Maroc corrige les erreurs de l'Algérie. Dans les tribus berbères on donne au droit berbère force de loi au même titre que le droit franco-berbère d'où l'arabe est proscrit, ainsi que l'enseignement religieux des écoles coraniques... L'évangélisation du Maroc est possible et nécessaire... C'est un axiome admis déjà par beaucoup mais que tout Français doit adopter : le Berbère sera assimilé qu'autant qu'il sera chrétien ».

De telles pages sont difficilement réfutables, encore qu'à aucun moment elles n'aient eu la moindre consécration officielle. Mais le fait qu'elles aient pu paraitre, et certaines sous des signatures connues, sans encourir la moindre censure, ne pouvait que fortifier l'idée qu'une «Croisade » était entreprise par les autorités françaises au Maroc, et c'est cet aspect du problème, inlassablement répété, qui sera au centre de la campagne de presse contre le Dahir que les nationalistes marocains entreprendront après le 16 mai. Quand on ajoute à ces éléments l'interdiction, ou tout du moins les empêchements, dont furent victimes les «fquihs" voulant se rendre dans les régions berbères, la réaction des Marocains se justifie pleinement.

Dans un tel contexte, la promulgation du Dahir Berbère permit aux Marocains d'exprimer leurs ressentiments et de manifester leur mécontentement. Est-ce à dire que la réaction fut immédiate et spontanée?

La chronologie ne semble pas le montrer. Bien au contraire, elle nous prouve, si besoin était, que les nationalistes marocains ont su admirablement exploiter l'erreur psychologique et politique que constituait la promulgation de ce dahir, pour exprimer leurs revendications sous couvert d'une défense de l'Islam, prétexte que leur offrait ce texte. Il est évident que le sort de leurs "frères » berbères tenait peu de place dans leurs préoccupations vu les appréciations fort peu amènes qu'ils portaient sur eux. En utilisant à des fins politiques un fait culturel indéniable - les différences entre Berbères et Arabes -, la France avait donné à de jeunes Marocains les moyens inespérés de se faire entendre par la totalité du monde Arabe. 


\section{Les Manifestations contre le Dahir Berbère}

La première manifestation populaire contre le Dahir Berbère eut lieu le 20 juin 1930 . C'est donc plus d'un mois après sa promulgation que les Marocains commencèrent à témoigner leur opposition à ce décret.

Que s'était-il passé entre temps?

C'est Abdellatif Sbihi, leader des "Jeunes Marocains de Salé ", qui fut à l'origine de la contestation. Totalement acquis aux idées nationalistes, Abdellatif Sbihi était traducteur dans l'administration quand le texte du Dahir lui parvint. Dès qu'il en eut compris la portée, il démissionna, et se rendit au collège Moulay Youssef à Rabat, où de nombreux jeunes Slaouis étudiaient. Là, il leur expliqua que par ce dahir, la France tentait de diviser territorialement le Maroc. Puis il effectua la même démarche à Fès, mais dut se rendre à l'évidence : l'explication, géographique et politique, qu'il donnait du dahir n'avait aucun impact sur les gens. Aussi commença-t-il à le leur présenter comme une atteinte à l'essence même de l'Islam.

9 Il fut aisé à Abdellatif Sbihi d'expliquer aux jeunes Lycéens de Rabat et de Salé les buts pernicieux du dahir. Un mercredi ils se réunirent et cherchèrent ensemble le moyen de réveiller une opinion apparemment assoupie, pour s'opposer à l'œuvre entreprise par la France. C'est Abdelkrim Hajji qui suggéra que la prière du «Latif » fût récitée à la mosquée. Cette prière n'était psalmodiée que pour demander l'aide de Dieu face à une calamité : inondation, sécheresse, invasion de sauterelles... durant des semaines, la prière du Latif fût entendue dans de nombreuses mosquées du Royaume, surtout à Fès, où la contestation fut la plus forte. Que ce soit à Fès, Salé, ou dans d'autres villes du Maroc, la mosquée était le lieu privilégié où pouvait s'exercer cette protestation, car il était le seul possible.

\section{La récitation des « Latifs » : juin-août 1930}

C'est à partir du 20 juin 1930 que la récitation des «Latifs » commença dans les grandes villes du Maroc. A Fès, elle fut quotidienne dans la mosquée de l'Université Qaraouiyne

52 Le 11 août eut lieu un événement particulier, sur lequel les nationalistes préféreront ne pas s'étendre. En effet, ce jour là, qui était le jour du Mouloud, une lettre du Sultan fut lue dans les mosquées de Fès, Rabat, Salé, Casablanca, Meknès et Marrakech.

Dans cette lettre le Sultan, Mohammed Ben Youssef, demandait l'arrêt des manifestations et justifiait la promulgation du Dahir Berbère...!

Que ce texte ait été rédigé par les services de la Résidence ne fait aucun doute, mais, tout comme pour le Dahir, le Sultan accepta de le signer et il fut lu en son nom. Après avoir rappelé les « coutumes ancestrales » qui, de tout temps avaient permis aux tribus berbères de régler leurs différends, le message du Sultan précisait que : «... L'exercice de ces coutumes s'est ainsi étendu sur plusieurs siècles et le dernier souverain qui l'a reconnu aux tribus berbères est notre auguste et vénéré père, qui n'a fait que suivre les traces de ses prédécesseurs, dans le seul but d'accorder aux Berbères le moyen de régler leurs différends pour le développement de la paix parmi eux. Cet octroi ne pouvant être considéré comme un moyen d'administration maghzenienne, nous avons nous-même décrété de semblables mesures par notre dahir chérifien. Cependant, des 
jeunes gens, dénués de toute espèce de discernement, ignorant toute la portée de leurs actes répréhensibles, se sont mis à faire croire que ces mesures que nous avons décrétées n'ont pour but que la christianisation des Berbères. Ils ont ainsi induit la foule en erreur et ont convié les gens à se réunir dans les mosquées pour réciter les prières du "Lactif" après les prières rituelles, transformant par ce procédé la prière en manifestation politique de nature à jeter le trouble dans les esprits.

Notre Majesté réprouve absolument que les mosquées dont Dieu a fait des lieux de prière et de piété, soient transformées en foyers de réunions politiques où prennent libre cours les arrière-pensées et où se développent les mauvais penchants ».

Seule concession faite aux protestataires, le Sultan accordait à toute tribu qui en exprimerait le désir la possibilité d'être soumise à la juridiction du Chrâa.

Il était plus difficile d'être plus ferme quant à la condamnation des manifestations.

Du 13 au 21 août, des négociations se tinrent entre Rabat et Fès, en vue de constituer la délégation qui viendrait présenter ses doléances au Sultan.

Le 23 août, 10 délégués furent choisis. Leur groupe prit le nom de Taifa, et, fait révélateur, chacun d'eux se donna le nom d'un des compagnons du Prophète. Que demandaient les protestataires?

1 - Le respect de l'autorité de Sa Majesté le Sultan, tant spirituelle que temporelle, ceci en obtenant que tous les agents du Maghzen ne soient responsables que devant le gouvernement chérifien.

2 - L'application de la loi religieuse par tous les tribunaux.

3 - L'unification des programmes d'enseignement, celui-ci ne devant être dispensé qu'en arabe, qui est la langue du Coran.

4 - Le respect de la langue arabe qui doit être celle de toute l'administration et des tribunaux dans tout le royaume, et donc, ne donner aucun caractère officiel aux dialectes berbères.

5 - L'arrêt du mouvement missionnaire et l'interdiction des déplacements de ses membres dans les tribus, les « souqs » et les «moussems ».

6 - L'arrêt des subventions venant du budget de l'Etat Chérifien aux associations missionnaires.

7 - Le refus d'autoriser la création par les missionnaires de nouveaux orphelinats et maisons d'accueil pour les enfants abandonnés. Ne pas subventionner ceux qui existent déjà, et faire en sorte que rien ne puisse demeurer sous la tutelle de ces missionnaires.

8 - L'interdiction pour tout prêtre ou missionnaire de diriger une école de l'Etat.

Ils demandaient aussi de ne pas entraver l'action des «fquih" dans les tribus pour qu'ils puissent enseigner les principes de la religion et inciter les gens à en respecter les règles, et donc de supprimer les « laisser-passer » à l'intérieur du Royaume. Enfin, ils réclamaient l'amnistie totale pour les emprisonnés et exilés qui l'avaient été en raison de leur participation aux événements récents.

Deux jours après, le 30 août au matin, la délégation reprenait la route de Fès, les mains vides, et faisait connaître aux Fassis, la réponse du Sultan : « ... Nous allons considérer votre demande et nous y répondrons ".

La décision fut alors prise de recommencer les manifestations, et des émissaires furent envoyés dans les grandes villes du pays, pour ranimer l'ardeur des nationalistes.

Mais les autorités du Protectorat étaient sur leurs gardes. Arrestations et exils furent plus sévères. Avec l'éloignement des principaux responsables de l'agitation, 
l'opposition au Dahir connut un répit certain, dû aussi à la reprise des cours dans les lycées et les Universités, à la fin de l'été 1930.

Il faut noter le rôle fondamental joué par la ville de Fès, et par son élite intellectuelle, composée aussi bien d'hommes formés à l'école traditionnelle, que par d'autres, plus jeunes, qui avaient bénéficié d'un enseignement beaucoup plus moderne. Les autres villes du Royaume ne semblent pas avoir été très touchées par le mouvement, à l'exception de Rabat et de Salé, qui furent en effervescence durant quelque temps. Il faut aussi noter le caractère politique des revendications marocaines sous couvert de la défense de l'Islam, ce qui était le seul moyen pour les nationalistes de provoquer le sursaut d'une masse de gens beaucoup trop préoccupés par la nécessité de gagner leur pain quotidien. Mais, outre le côté affectif, le nationalisme, en s'appuyant sur les mosquées, auréolait son combat d'un caractère sacré. De même, en prenant le nom des compagnons du Prophète, les principaux chefs adoptaient la structure d'une confrérie. Non seulement le mouvement nationaliste puisait dans l'Islam le moteur de ses revendications, mais il retrouvait aussi les moyens d'action et l'organisation qui avaient permis son expansion. C'était l'impact d'un fait sociologique séculaire sur un phénomène récent qui était le nationalisme.

\section{Les Réactions des Autorités Françaises}

Parallèlement aux réactions locales et ponctuelles que nous avons déjà citées: emprisonnements, exils, brutalités, visant à faire cesser toute manifestation, les autorités françaises firent paraître une série d'articles expliquant et justifiant la politique berbère de la France, qui furent publiés dans le Bulletin du Comité de l'Afrique Française.

Bien que l'agitation eût cessé, les esprits restaient tendus, et, dans un but d'apaisement, le Président de la République Française, Gaston Doumergue, se rendit au Maroc du 14 au 24 octobre 1930.

Peu de jours auparavant, le 6 octobre, une circulaire du Résident Général, donnait des instructions très nettes concernant le prosélytisme fort peu discret des missionnaires chrétiens. C'était admettre que les protestations des Marocains étaient fondées, et qu'il fallait mettre un terme à leurs critiques. Les mesures prises à l'encontre des manifestants firent cesser l'agitation à l'intérieur du Maroc, mais elle continua, et même s'amplifia, en France et au Moyen-Orient, par une campagne de presse dont certains articles furent très violents et même tendancieux.

\section{La Campagne à l'extérieur du Maroc}

Cette violente campagne de presse fut organisée par de jeunes nationalistes, ceux-là mêmes qui avaient été parmi les premiers à protester au Maroc contre le Dahir. C'est ainsi que Ahmed Balafrej, Mekki Naciri, Mohammed El Fassi, Abdelqader Benjeloun, Mohammed Kholti, Abdelmalek Faraj et Mohammed Ouazzani, conseillés par Chakib Arslan, envoyèrent, de Paris le plus souvent, une série d'articles qui furent publiés par la presse du Caire.

En France, dès le début de l'année 1931, ils publièrent, sous le nom de «Mouslim 
Barbari » un opuscule intitulé Tempête sur le Maroc, avant de fonder à Paris, en 1932, la revue Maghreb.

La caractéristique essentielle de cette campagne est la dramatisation consciente du contenu du Dahir, présenté essentiellement comme une tentative de christianisation des Berbères, qui formaient la majeure partie de la population du Maroc. Il fallait toucher les sentiments profonds de tous les Musulmans du monde, et, dans le contexte psychologique et politique du moment, présenter le dahir comme une attaque contre l'Islam, ce qui ne pouvait que provoquer une levée de boucliers. Mais, derrière la défense de leur religion, c'était aussi l'action de la France qui était la cible de toutes les critiques des nationalistes, le dahir étant plus que jamais un prétexte. Il était évident qu'une telle dramatisation avait pour corollaire une exagération très tendancieuse des faits, et que l'honnêteté intellectuelle ne fut pas la principale qualité de cette campagne.

\section{Mouslim Barbari}

Début 1931, paraissait à Paris un opuscule polémique Tempête sur le Maroc, ou les erreurs d'une Politique Berbère, dont l'auteur était «Mouslim Barbari ». En fait, derrière ce pseudonyme, on trouve les plumes de Ahmed Balafrej, Mohammed El Fassi, Abdelqader Benjeloun, Mohammed Ouazzani... ainsi que celle du journaliste français Daniel Guérin.

Déjà, le $1^{\text {er }}$ novembre 1930, le journal Le Monde publiait un article de Guérin intitulé « La France règne au Maroc ", dans lequel l'auteur reprenait l'argument principal de toute la campagne de protestation contre le dahir : «... La France qui se prétend une grande puissance musulmane, est tout simplement en train de tenter de désislamiser les Berbères du Maroc ». En voulant : « ... diviser pour régner, l'impérialisme français avait déchaîné une véritable tempête sur le Maroc ».

Mouslim Barbari va donc décrire cette « tempête », en reprenant le grief, constamment formulé à l'encontre de la politique berbère: la volonté des autorités françaises de «christianiser et d'évangéliser » les Berbères.

Mais c'est au Moyen-Orient que, orchestrées par Chakib Arslan, les protestations les plus violentes, mais aussi les plus tendancieuses contre le Dahir Berbère se feront entendre, et cela, dès Octobre 1930.

En mars 1930 Chakib Arslan fait paraître à Genève, avec l'aide de Ihsan Bey El Jabri, la revue La Nation Arabe centrée sur la défense de l'Islam dans le monde. C'est au nom de l'Islam " persécuté » qu'il intervient en 1930 contre le Dahir Berbère, dans une série d'articles dont les termes sont ceux que nous connaissons, l'idée essentielle étant que la France, en créant des tribunaux coutumiers "primitifs et de basse qualité ", voulait affaiblir les Musulmans en les divisant pour consolider sa domination. Auparavant, Arslan avait fait un court séjour au Maroc, très peu de temps après la promulgation du Dahir. Parti de Paris, il s'était rendu à Tanger, en passant par Madrid, où il avait été rejoint par Ahmed Balafrej et Mohammed El Fassi. Il arriva à Tanger le 9 août 1930, mais il en fut immédiatement expulsé par les autorités françaises. Il se rendit alors à Tétouan, où il resta 10 jours. Il eut de nombreux contacts avec les nationalistes marocains dont Abdelhaq Torres et Abdeslam Bennouna. C'est après son séjour à Tétouan qu'il adressa à l'ensemble du monde musulman une lettre par laquelle il exhortait les Arabes à protester contre le dahir: «... Il faut que tous les journaux 
musulmans protestent de la façon la plus véhémente en Egypte, en Palestine, en Irak, en Syrie, aux Indes et à Java ». Cet appel fut entendu.

Au Moyen-Orient, c'est, parmi d'autres, le journal El Fath dirigé par Muhib Ed Din Khatib qui fut le vecteur de la campagne contre le dahir. Les articles, souvent non signés pour des raisons de sécurité, étaient rédigés par des nationalistes marocains, conseillés par Arslan. Il faut noter que le correspondant de ce journal à Alexandrie n'était autre que Hassan El Banna, fondateur, en 1928, de la célèbre «Association des Frères Musulmans ».

Ancien rédacteur du prestigieux quotidien Al Ahram, Muhib Ed Din Khatib dirigea l'imprimerie Salafiya qui fit paraitre en février 1917 sa première revue, la « Majalla Salafiya ", destinée à propager les idées du mouvement salafiste. D'autres publications s'ajoutèrent, dont le but essentiel était de réunir les Musulmans autour d'un thème central, qui était la défense de l'Islam, partout où il était attaqué. Il était aussi le secrétaire d'une revue mensuelle Majallat al Shuban Al Muslimin, organe d'une société littéraire et religieuse Jamiyat Al Shuban Al Muslimin (la Société des Jeunes Musulmans), fondée en 1927, dont les buts étaient la diffusion des principes moraux de l'Islam et la lutte contre tout ce qui pouvait diviser les musulmans. Mohib Ed Din Khatib centralisa toutes les informations venant du Maroc, et les publia dans les journaux dont il était le directeur, assorties de commentaires, dont le moins que l'on puisse dire est que l'honnêteté intellectuelle n'était pas leur qualité principale.

C'est ainsi que la politique de la Fance est assimilée à une «croisade » menée par des "milliers de missionnaires ", bien déterminés à combattre le Foi Musulmane par tous les moyens. Pour atteindre ces buts, ils ont à leur disposition des " sommes énormes ", allouées par l'Etat, mais ils perçoivent aussi, ce qui est "scandaleux», des subsides provenant des Biens Habous, alors que cet argent est destiné aux fondations pieuses de l'Islam. Ils ont de plus la caution morale du Résident Général qui œuvre pour que le Maroc émerge « de la longue nuit de l'Islam ». C'était une " christianisation par le fer et par le feu » qui était imposée aux gens, c'était à nouveau le temps de l'Inquisition, avec son cortège d'iniquités et d'horreurs...!

Mais, au pied de l'Atlas, si «le Lion dort», il ne faut pas désespérer car un jour, il se réveillera, et sa vengeance sera terrible...

Bien que fort tapageuse et tendancieuse, affirmant d'une façon péremptoire des faits totalement faux ou invérifiables, répétant à longueur de colonnes les mêmes arguments, cette campagne de presse eut une efficacité indéniable. Elle se calma durant l'année 1931, mais retrouva une certaine vigueur en Mai 1932 et 1933, dates anniversaires de la promulgation du dahir. Cette campagne réussit à faire l'unanimité des pays musulmans contre la politique indigène de la France, ce qui amena les autorités du Protectorat à reconsidérer certains aspects de cette politique.

\section{Vers le Dahir du 8 avril 1934}

Bien qu'elles fissent la sourde oreille devant les protestations qui émanaient de tout le monde arabe, les autorités du Protectorat ne pouvaient cacher leur embarras et leur impuissance à arrêter cette campagne. Elles ne pouvaient ignorer la situation et tentèrent $d$ 'y apporter une solution, mais sans pour autant se déjuger. Il faudra attendre 1932 pour que paraisse dans le B.C.A.F. un article très critique sur le 
fonctionnement de la justice indigène, dont l'auteur était un juriste: Henri Bruno. L'auteur n'hésitait pas à critiquer la façon dont était appliquée la loi religieuse par des cadis: «recrutés au petit bonheur, sans garantie sérieuse de moralité ni de compétence ». La justice civile, elle, est rendue par des pachas et des caïds souvent « illettrés » dont les caractéristiques sont l'arbitraire et la vénalité. Quant à la justice coutumière berbère, deux ans après le dahir du 16 mai, elle n'est pas encore dotée des tribunaux coutumiers d'appel, et les tribunaux français qui - d'après le dahir -étaient habilités à juger des crimes commis en pays berbère, n'étaient saisis que d'une façon exceptionnelle. Il fallait donc procéder à une révision de cette justice, ne serait-ce que pour ne plus prêter le flanc à la critique. C'est pourquoi, en mars 1933, on avait pensé remanier la justice berbère dans le cadre plus général d'une réorganisation totale de la justice indigène, mais une telle entreprise risquait d'être perçue comme un désaveu de l'œuvre du Résident Général. Or, en juillet de cette même année, Lucien Saint fut remplacé par Henri Ponsot. Le nouveau Résident Général avait les mains libres pour entreprendre la réforme nécessaire de la justice indigène. Dès l'arrivée du Nouveau Résident, un article du B.C.A.F préparait ses lecteurs à cette réforme. Trois ans après les textes signés Le Glay, Mohand ou Aqqa, il était indéniable que les autorités françaises avaient changé d'optique. L'auteur, M.D. Troyes, mettait l'accent sur la nécessité de reprendre l'examen de la justice berbère, en se plaçant au point de vue des réactions politiques qu'il avait suscitées. En premier lieu il fallait reconnaître que le désarroi des esprits dans le monde musulman était fondé, malgré les "exagérations et les calomnies » accumulées dans la presse arabe. De plus, laisser croire que le dahir était un moyen de séparer un "bloc berbère" du "bloc arabe " relevait de l'utopie, à laquelle venaient s'ajouter des "imprudences de langage", dûes à certaines publications catholiques. En conséquence conclut l'auteur, s'il est absolument exclu de revenir sur les juridictions coutumières et de laisser le Maghzen imposer le Chrâa, par contre il n'est pas exclu de reconsidérer la formulation de l'article 6 du dahir, ce qui fut réalisé par le dahir du 8 avril 1934.

Ce dahir uniformisait, en matière pénale, les juridictions des Pachas et des Caïds, et donnait au Haut Tribunal Chérifien toute compétence pour juger des crimes commis en pays de coutume.

C'était l'abrogation de l'article 6 du 16 mai 1930.

Le texte en exposait les motifs dans les termes suivants :

«Dans le but d'améliorer les conditions de fonctionnement de la justice Maghzen, l'utilité Nous est apparue, d'une part, de soumettre à un régime uniforme, en matière pénale, toutes les juridictions de pachas et caïds et, d'autre part d'étendre à Notre Empire la compétence du Haut Tribunal Chérifien, pour la répression des infractions prévues à l'article $1^{\text {er }}$ du dahir du 4 août 1918..."

Notre Majesté Chérifienne a décidé ce qui suit :

Art. $1^{\text {er }}$ :Les règles de compétence et de procédure du Dahir du 4 août 1918 sont applicables, en matière pénale et dans toute l'étendue de l'Empire Chérifien, aux mahakmas des Pachas et Caïds qui fonctionnent sans l'assistance d'un Commissaire du Gouvernement, les attributions de cet agent étant remplies par l'autorité de contrôle.

La compétence dévolue au Haut Tribunal Chérifien par le dahir du 4 août 1918 est étendue, en toutes matières criminelles, aux pays de coutume de Notre Empire.

Art. 2 : Il est créé, au Haut Tribunal Chérifien, une section pénale coutumière chargée de connaître dans les conditions prévues par les dahirs du 4 août 1918, tant des appels interjetés contre les jugements rendus en matière pénale par les pachas et caïds des 
tribus de coutume, que des infractions commises par Nos sujets dans ces mêmes tribus, et prévues à l'art. $1^{\text {er }}$ du dahir du 4 août 1918.

\section{Conclusion}

Par le Dahir du 16 mai 1930, en voulant entériner en droit ce qui existait en fait, les Autorités Françaises du Protectorat avaient commis une erreur psychologique dont les conséquences politiques dépassèrent, et de loin, ce qu'il eût été normal d'en attendre. Certes, le recul de la Résidence, par la promulgation du Dahir du 8 avril 1934 qui abrogeait l'article 6 du Dahir du 16 mai 1930, prouvait que la campagne de protestations avait porté ses fruits. Mais, contrairement à ce que certains historiens ont pu prétendre, cela ne signifiait en rien la fin de la politique berbère du Protectorat. Bien au contraire.

En effet, les tribunaux coutumiers continuèrent à fonctionner, leur nombre augmenta, et il en fut de même pour les écoles franco-berbères dont l'influence grandit auprès des populations locales. Quant aux tribus de coutume, elles furent de plus en plus nombreuses à être régies par leur Orf, surtout après la reddition des Ait 'Atta qui résistaient encore dans le Sagrho, et en 1941, cinq nouvelles tribus étaient classées, par arrêté viziriel, parmi celles dans lesquelles l'Orf serait appliqué. Il serait donc exagéré de penser que l'échec - fort relatif - du Dahir Berbère ait eu des conséquences importantes quant à la poursuite de la politique de la France dans les tribus de coutume. Par contre, l'importance de cet épisode de la politique berbère du Protectorat réside essentiellement dans une prise de conscience par le mouvement nationaliste de son unité, fortifiée dans la lutte qu'il mena contre le Dahir. Durant ces quelques années, "Vieux Turbans » et "Jeunes Tarbouches" virent leurs appréhensions et préjugés disparaître au profit d'une action, d'abord réformiste, puis très vite politique. Si la défense de l'Islam restera un principe fondamental de cette lutte, elle s'élargira à toute la société, et le $1^{\mathrm{er}}$ Décembre 1934, le Comité d'Action Marocaine ouvrira une nouvelle phase de l'histoire du Maroc.

\section{BIBLIOGRAPHIE}

ABU-NASR Jamil M., « Salafiya (The) Movement in Marocco : the religious Bases of Maroccan nationalist Movement ». St Antony's papers, XVI, 1965, pp. 90-103.

AGERON Charles-Robert, Politiques coloniales au Maghreb. P.U.F, 1972, 288 p. (Coll. Hier).

AUBIN Eugène, Maroc (Le) d'aujourd'hui. Paris, A. Colin, 1913.

BARBARI Mouslim, Tempête sur le Maroc, ou les erreurs d'une « politique berbère ». Paris, Ed. Rieder, 1931.

BESSIS Juliette, « Chekib Arslan et les mouvements nationalistes au Maghreb ». Rev. Hist. (CLIX) 2, 1978, pp. 467-489. 
BIDWELL Robin, Morocco under Colonial Rule. French administration of Tribal Areas, 1912-1956. London, Frank Cass, 1973, XVI-349 p.

BRown Kenneth, « The impact of the Dahir Berbère in Salé » in Gellner-Micaud, Arabs \& Berbers, $p$. 201-215.

LAFUENTE Gilles, « Dossier marocain sur le dahir berbère de 1930 », ROMM, 38 (2), 1984, p. 83-116.

LUCCIBONI Joseph, « L'élaboration du dahir berbère du 16 mai 1930 », ROMM, 38 (2), 1984, p. 75-81.

MADARIAGA M. R. de, « Le Dahir berbère de 1930 et la Société des Nations », Cahiers de la Méditerranée, 19, 1979, p. 58-128.

INDEX

Mots-clés : Histoire contemporaine 